\title{
AUTONOMOUS MICROSYSTEMS FOR ASTROBIOLOGY: DEVELOPMENT OF, AND SPACEFLIGHT RESULTS FROM, THE O/OREOS NANOSATELLITE
}

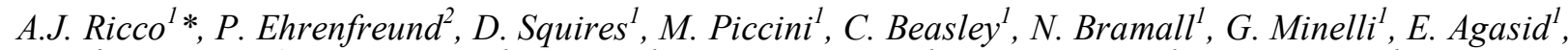

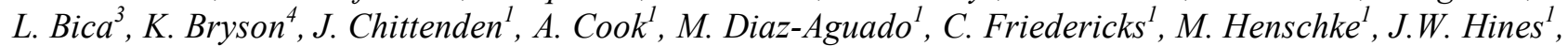

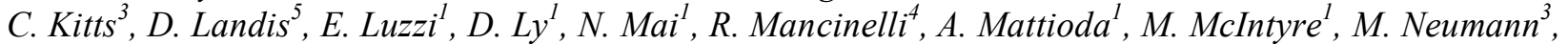

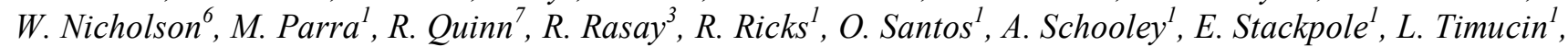 \\ B. Yost ${ }^{1}$, and A. Young ${ }^{3}$ \\ ${ }^{1}$ NASA Ames Research Center, Moffett Field, California, USA \\ ${ }^{2}$ Space Policy Institute, Washington, DC, USA \\ ${ }^{3}$ Robotic Systems Laboratory, Santa Clara University, Santa Clara, California, USA \\ ${ }^{4}$ Bay Area Environmental Research Institute, Sonoma, California, USA \\ ${ }^{5}$ Draper Laboratory, Cambridge, Massachusetts, USA \\ ${ }^{6}$ University of Florida, Kennedy Space Center, Florida, USA \\ ${ }^{7}$ SETI Institute, Mountain View, California, USA
}

\begin{abstract}
We designed, developed, constructed, tested, launched, and are now analyzing data telemetered from orbit by the O/OREOS (Organism/ Organic Exposure to Orbital Stresses) nanosatellite. Measuring $\sim 10 \times 10 \times 34 \mathrm{~cm}$ and weighing $5.5 \mathrm{~kg}$, O/OREOS (Figure 1) launched in November 2010 into 650-km Earth orbit as a secondary payload from Kodiak, Alaska. O/OREOS comprises three conjoined $10-\mathrm{cm}$-cubes, each a fully integrated microsystem: a "bus" including communications, power, and control functions; the Space Environment Survivability of Living Organisms (SESLO) experiment, and the Space Environment Viability of Organics (SEVO) experiment. We will present results from SEVO's real-time analysis of the photostability of organics and biomarkers over space exposure times of one year, and SESLO's data on the survival and metabolic activity of microorganisms at 3 timepoints spanning 6 months.
\end{abstract}

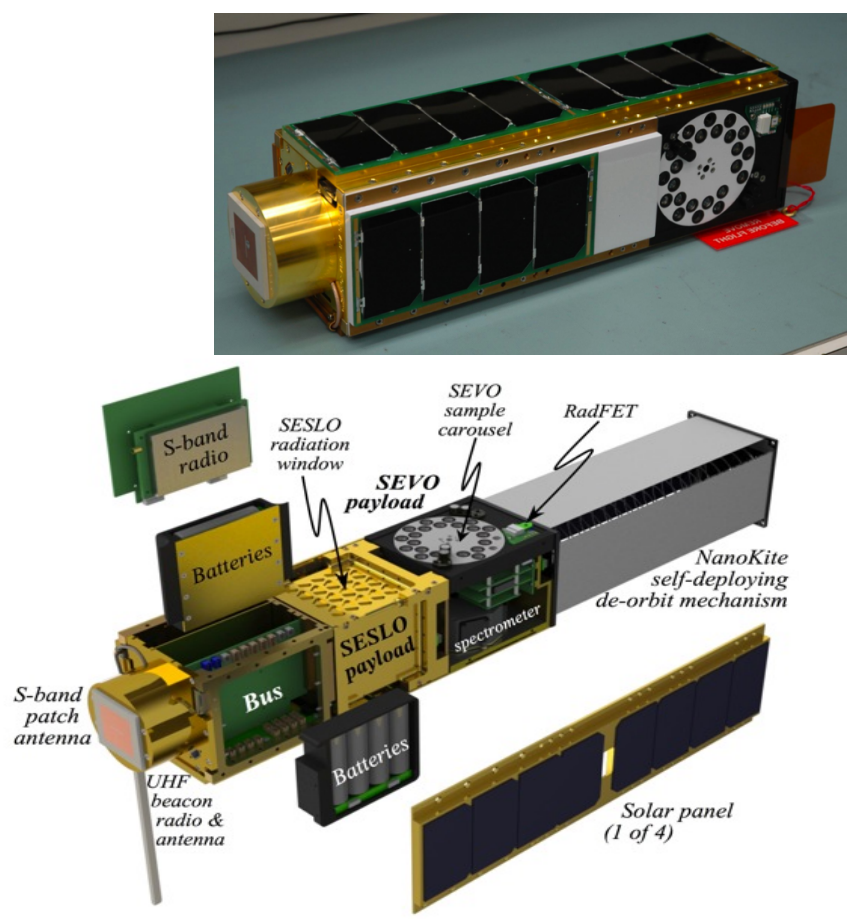

Figure 1: Photograph (top) and exploded solid model (bottom) of O/OREOS nanosatellite (10 × $10 \times 34 \mathrm{~cm}$, excluding nanokite).
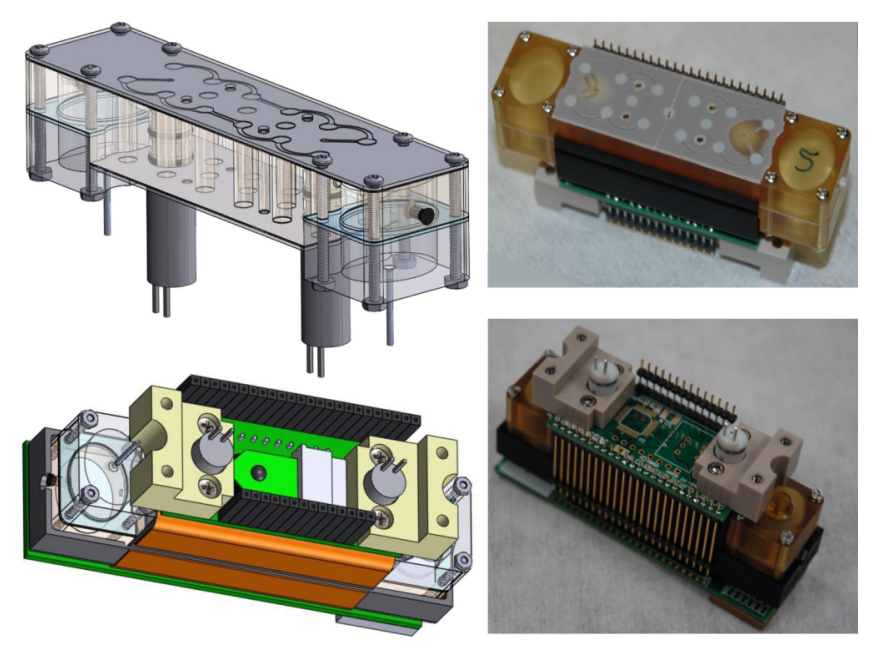

Figure 2: Four different views (solid model at left, photographs at right) of one of three bioblocks included in the SESLO payload. The two solenoid valves protrude down from the block (upper left); reservoirs are the elliptical structures at both ends of the block; heater is orange; radiation-sensitive FET (RadFET), which measures integrated radiation dose at two locations in each of the two payload cubes, is a black dot near the center of the bottom left.

\section{INTRODUCTION}

Small autonomous satellites under $10 \mathrm{~kg}$, referred to as nanosatellites or cubesats, can simultaneously reduce the cost and increase the accessibility of space science experiments [1]. Leveraging and integrating recent advances in nano-, micro-, and miniature technologies in fields from biotechnology to materials to sensors to telecommunications, cube/nanosatellites are under development by nearly 100 universities, numerous small commercial ventures, large aerospace companies, a dozen developing nations, and the world's major space agencies. Despite their small size, such spacecraft can support complex science, as in our drug-dosedependence experiments aboard PharmaSat [2]. The coupling of autonomy and telemetry in these orbital "free flyers" can provide near-real-time kinetic data as space science experiments progress.

Astrobiology seeks understanding of the origins, evolution, development, and future of life in the universe. O/OREOS addresses this by characterizing the space environment degradation of organic starting materials for life and biomarkers, and by probHilton Head Island, South Carolina, June 3-7, 2012 
ing the details of microorganism survival under the influence of microgravity and ionizing radiation. It also supports NASA's scientific exploration program by investigating Earth's local space environment and space biological effects relevant to future lunar and Mars missions.
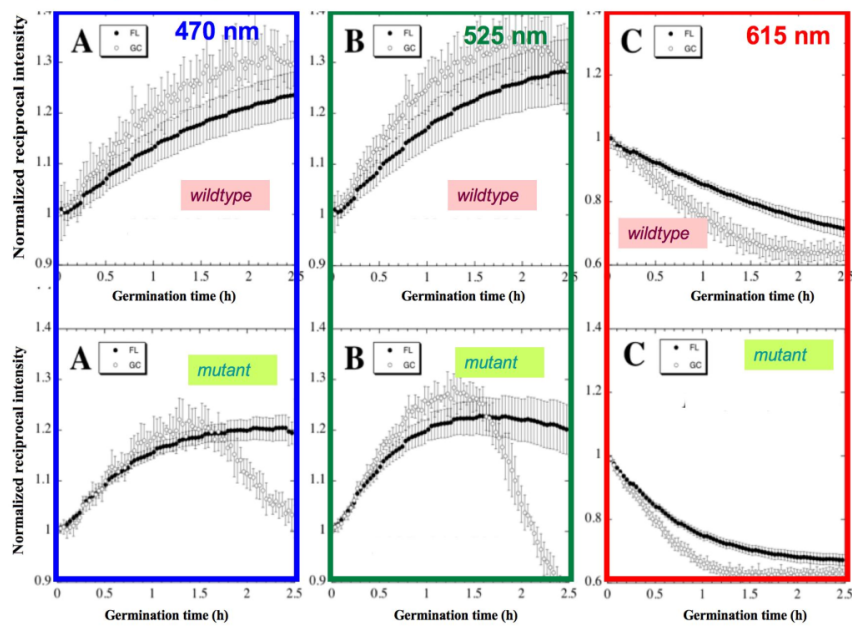

Figure 3: Growth curves (as normalized reciprocal intensity) after 14 days in space (solid dots) and an identical ground control (open circles) for B. subtilis wildtype and mutant strains at three wavelengths. Error bars are for $4-6$ wells of each strain of the microbe. The data show that growth is somewhat slower in the microgravity environment of space due to differences in mass transport of nutrients and waste products.

\section{DETAILS, RESULTS, AND DISCUSSION}

Soon after its orbital deployment and then after 3 and 6 months in space, SESLO characterized the growth, activity, health and adaptation of microorganisms to the stresses of outer space. SESLO consists of three miniature "bioblock" modules, each made by a combination of miniature machining and thin-film processing to include twelve $75-\mu \mathrm{L}$ sample wells plus a pair of valves and reservoirs (Figure 2). Groups of 6 wells are microfluidically connected via a solenoid valve to a reservoir containing growth medium the microbes.

Absorbance through each $2.8 \times 12 \mathrm{~mm}$ well was monitored using 3-color (RGB) LED illumination and an intensity-tofrequency detector. Prior to spaceflight, microbes were dried onto microwell walls and bioblocks sealed with gas-permeable mem-
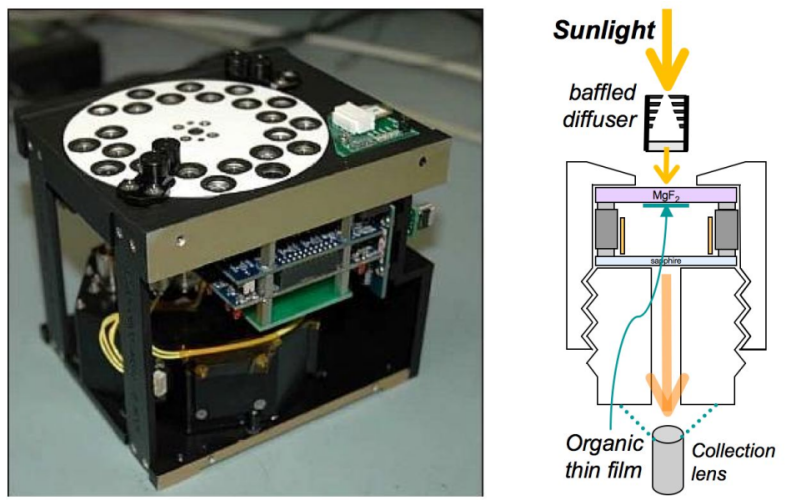

Figure 4: SEVO spectrometer payload cube (left) and cross sectional diagram of one of the 24 SEVO sample cells (right), $1 \mathrm{~cm}$ in diameter. $\mathrm{MgF}_{2}$ optical flat (at top of cell) transmits solar radiation to vacuum UV wavelengths, including the Lyman alpha hydrogen emission at $124 \mathrm{~nm}$. branes. The growth medium included the viability dye Alamar blue, which changes color due to cellular metabolic activity. Figure 3 shows initial growth curves telemetered from orbit [3].

SEVO tracked the outer space photostability of organics; solar and space radiation are attenuated only by $1.5-\mathrm{mm} \mathrm{MgF}_{2}$ windows that support thin-film samples. Four molecular classespolycyclic aromatic hydrocarbon, amino acid, quinone, metalloporphyrin-represent "bio-building-blocks" and biomarkers. SEVO comprises a miniaturized UV-visible-NIR spectrometer and a 24-sample carousel that holds hermetically sealed microcells (Figure 4). Integral optics enable the use of the Sun as the light source. After transiting a given organic film, light travels via optical fiber to the spectrometer $(<2 \mathrm{~nm}$ spectral resolution; $200-$ $1000 \mathrm{~nm}) .16$ spectral acquisitions are averaged and stored biweekly for each cell, then telemetered. Figure 5 shows a SEVOacquired solar reference spectrum. Details of the spectroscopically-measured chemical changes of organic thin films resulting from over one year of full solar exposure in orbit will be reported in a forthcoming publication [4].

\section{CONCLUSIONS}

The success of the O/OREOS mission demonstrates the utility of low-cost nanosats as space science research and technology development platforms that can capitalize on the latest technologies to fly instruments that are truly state of the art. The O/OREOS Mission was supported by the NASA Astrobiology Small Payloads Program and Planetary Protection Office.

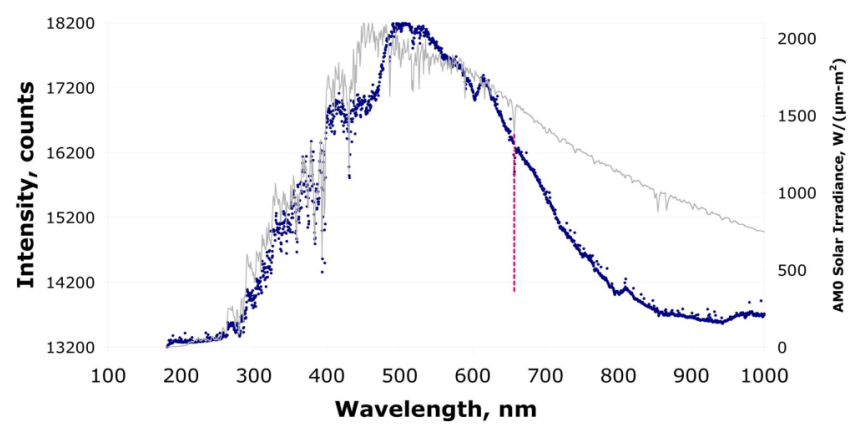

Figure 5: Solar spectrum measured at an orbital altitude of 650 $\mathrm{km}$ by the SEVO spectrometer (dots, left axis) compared to a reference solar spectrum (gray line, right axis) showing excellent alignment of spectral features. Organic thin film absorbances are measured using the sun as the light source for the spectrometer.

\section{REFERENCES}

[1] K. Woellert, P. Ehrenfreund, et al., "Cubesats: Cost-Effective Science and Technology Platforms for Developing Nations," Adv. Space Res., 47, 663 (2011).

[2] A.J. Ricco, M. Parra et al., "PharmaSat: Drug Dose Dependence Results from An Autonomous Microsystem-Based Small Satellite," Tech. Digest 2010 Solid-State Sensors, Actuators, and Microsystems Workshop, Transducer Research Foundation: San Diego (2010); pp. 110-113.

[3] W.L. Nicholson, A.J. Ricco, et al., "The O/OREOS Mission: First Science Data from the SESLO Payload," Astrobiology, 11, 951-958 (2011).

[4] A. Mattioda, A. Cook, et al., "The O/OREOS Mission: First Science Data from the Space Environment Viability of Organics (SEVO) Payload," Astrobiology, in preparation (2012).

\section{CONTACT}

*A.J. Ricco, tel: +1-650-604-4276; ajricco@stanford.edu 